\title{
Paradoxical Cerebral Embolism Secondary to Superior Vena Cava Occlusion and Development of a Systemic Right-to-Left Shunt
}

\author{
Ronny J.D. Kuang ${ }^{1}$ Anna L Beale $e^{2,3, \odot}$ \\ ${ }^{1}$ Department of Radiology, Alfred Hospital, Melbourne, Victoria, \\ Australia \\ ${ }^{2}$ Department of Medicine, Monash University, Melbourne, Victoria, \\ Australia \\ ${ }^{3}$ Department of Cardiology, Alfred Hospital, Melbourne, Victoria, \\ Australia \\ ${ }^{4}$ Department of Surgery, Monash University, Melbourne, Victoria, \\ Australia \\ ${ }^{5}$ National Trauma Research Institute, Melbourne, Victoria, Australia
}

Jason E Bloom 2,3

Warren Clements ${ }^{1,4,5, \odot}$

Address for correspondence Warren Clements, BBiomedSc (Hons), MBBS, FRANZCR, EBIR, Department of Radiology, Alfred Health, 55 Commercial Road, Melbourne, VIC 3004, Australia (e-mail: w.clements@alfred.org.au).

\begin{abstract}
Keywords

- superior vena cava obstruction

- right-to-left shunt

- embolization

Paradoxical cerebral embolism, although rare, can be secondary to acquired causes such as superior vena cava (SVC) occlusion and development of a mediastinal right-to-left shunt. Such shunts allow undisturbed passage of thromboemboli to bypass the pulmonary circulation and enter the systemic circulation. This report presents a case of paradoxical cerebral embolism due to a right-to-left shunt originating from occlusion of the SVC. The etiology of the SVC occlusion stems from a prior central venous access line used for treatment of lymphoma. The patient underwent endovascular treatment with successful coil embolization of the mediastinal shunt.
\end{abstract}

\section{Background}

Superior vena cava (SVC) obstruction most often occurs in the context of metastatic pulmonary or mediastinal malignancy but can also be seen secondary to infection, idiopathic mediastinal fibrosis, benign tumors, mediastinal hematoma, radiation fibrosis, or iatrogenic causes. ${ }^{1-5}$ Obstruction secondary to the presence of a central venous access device is rare, but a recognized complication. Over time, mediastinal shunts can develop allowing for the formation of collateral pathways between the pulmonary and systemic circulation. This report presents a case of paradoxical cerebral embolism from a right-to-left fistula and the subsequent endovascular treatment.

\section{Case Presentation}

A 51-year-old woman presented to the emergency department with a history of sudden-onset aphasia and confusion. However, by the time she arrived at the hospital 6 hours later, most of her neurological symptoms had resolved with mild residual dysphasia. No associated limb weakness, cranial nerve dysfunction, or posterior circulation symptoms were found on examination. Her past medical history included Hodgkin's lymphoma (remission) that was treated with a stem cell transplant three years ago.

Initial investigations included a computed tomography (CT) scan of the brain and a CT angiogram of the head and neck which demonstrated a suspected area of subacute infarct published online May 18, 2021
DOI https://doi.org/

$10.1055 / \mathrm{s}-0041-1730104$ ISSN 2457-0214 (c) 2021. Indian Society of Vascular and Interventional Radiology. This is an open access article published by Thieme under the terms of the Creative Commons Attribution-NonDerivative-NonCommercial-License, permitting copying and reproduction so long as the original work is given appropriate credit. Contents may not be used for commercial purposes, or adapted, remixed, transformed or built upon. (https://creativecommons.org/licenses/by-nc-nd/4.0/). Thieme Medical and Scientific Publishers Pvt. Ltd. A-12, 2nd Floor, Sector 2, Noida-201301 UP, India 
in the left parietal lobe. No carotid stenosis was identified and extended cardiac telemetry did not demonstrate atrial fibrillation as an embolic source. Follow up magnetic resonance imaging (MRI) brain confirmed this finding with evidence of acute bilateral parietal lobe infarcts ( - Fig. 1). A central source of thromboembolism was suspected and transthoracic echocardiogram (TTE) with intravenous (IV) saline/bubble study was performed, revealing systemic venous to pulmonary venous shunting. Contrast was seen entering the left atrium without transitioning through the right atrium (-Fig. 2). CT venogram of the chest validated these findings showing a chronic long-segment occlusion of the SVC including the azygous vein tributary. A large shunt/fistula was seen to drain into the right superior pulmonary vein ( - Fig. $\mathbf{3 a}, \mathbf{b}$ ). In addition, there were numerous collateral pathways including smaller mediastinal right-right shunts which were bypassing the occluded SVC/azygous and draining into the Inferior Vena Cava (IVC) or directly to the right atrium. The cause of the SVC occlusion was felt most likely due to a previous central venous access device (CVAD) as the patient had an implantable port for 2 years and history of previous temporary central line insertions, used during treatment of lymphoma.

The patient made a full recovery and was discharged home on apixaban $5 \mathrm{mg}$ daily. After discussion at a multidisciplinary meeting, the patient opted to proceed to have an angiographic study of her chest with embolization of her right-to-left shunt.

Under conscious sedation (midazolam and fentanyl), the right brachial vein was accessed using a modified Seldinger

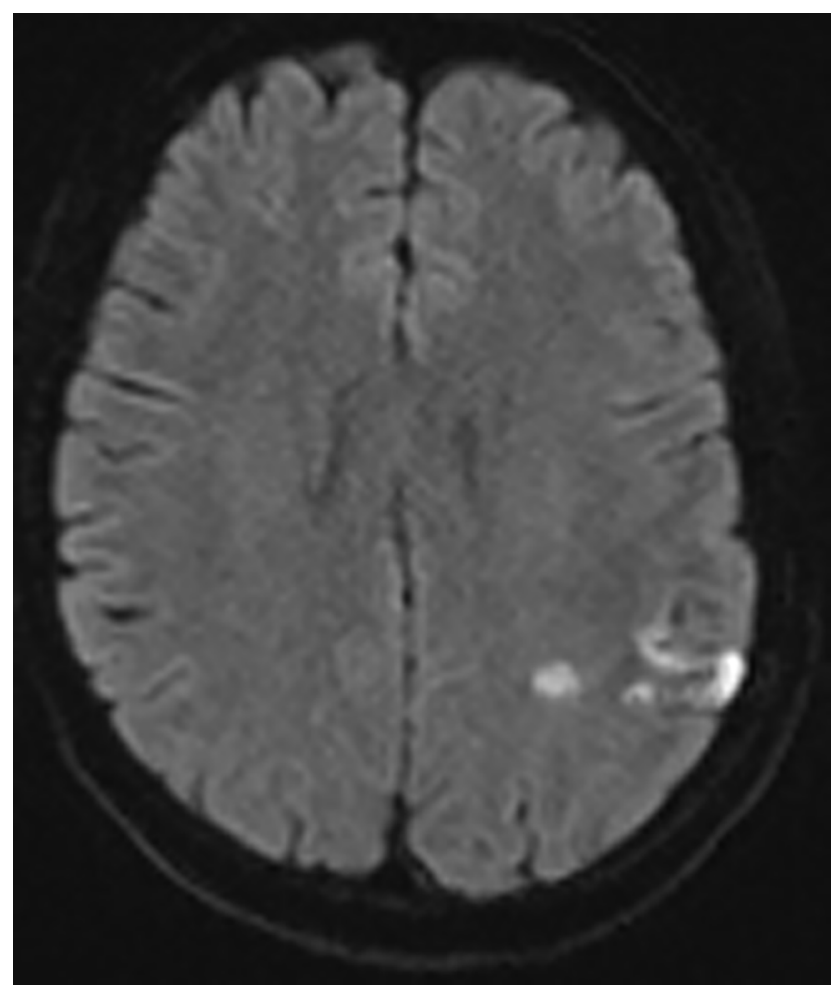

Fig. 1 Diffusion-weighted imaging of the superior cerebral hemispheres demonstrates punctate and curvilinear areas of abnormal diffusion restriction affecting the left parietal lobe and adjacent deep cerebral white matter. Findings were in keeping with areas of acute cerebral infarction.

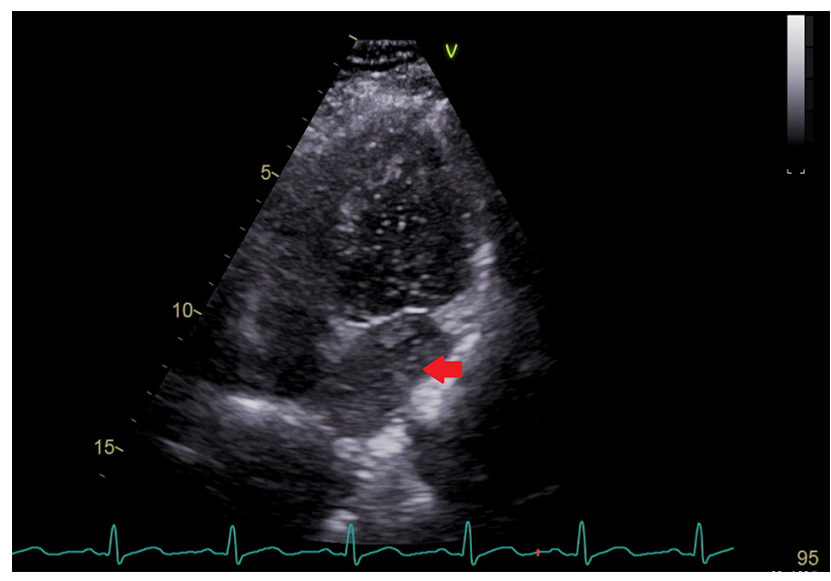

Fig. 2 Transthoracic echocardiogram demonstrating agitated saline contrast, injected via the right cubital fossa, opacifying the left atrium (arrow) without transit through the right heart and pulmonary circulation first.
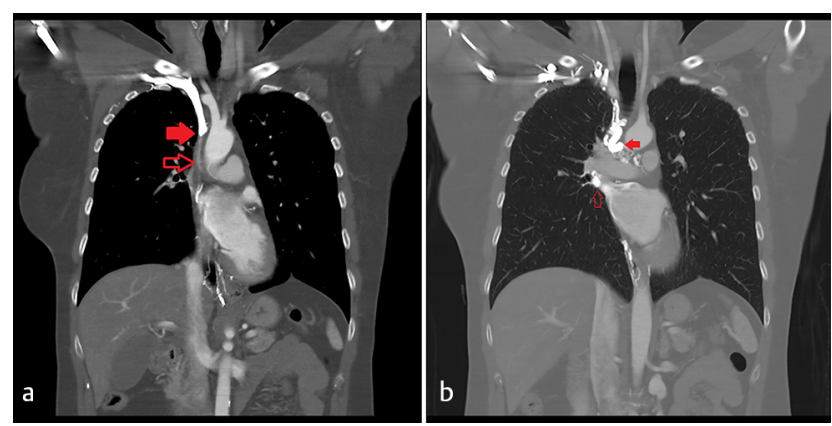

Fig. 3 Coronal computed tomography (CT) scan of chest with first pass venogram via the right arm. (a) There is abrupt cutoff of the superior vena cava (SVC, arrow) at the level of the great vessels containing dense contrast media. Immediately below this, the SVC is a thick cord like structure confirming longstanding occlusion (open arrow). (b) Large contrast-filled collateral vessels are present in the mediastinum with a large right-to-left shunt (arrow) returning venous circulation into the left superior pulmonary vein (open arrow).

technique and micropuncture access kit (Cook Medical, Bloomington, Indiana) and a 6-French sheath $(45 \mathrm{~cm}$; Destination, Terumo, Japan) inserted. Intravenous unfractionated heparin was administered (5,000 units). An angle tapered catheter (65 cm; Kumpe, Angiodynamics, Latham, New York) was used to select the shunt and angiography performed. This demonstrated occlusion of the SVC with a primary large shunt/fistula draining to the right superior pulmonary vein with early filling of the left atrium ( - Fig. 4). Numerous small right-to-right shunts were also noted draining to the right atrium. The largest right-to-left shunt was selected with a microcatheter (2.7 French $135 \mathrm{~cm}$; Progreat, Terumo, Japan) and embolized initially with $8 \mathrm{~mm}$ and $6 \mathrm{~mm}$ detachable coils (Ruby coil, Penumbra Inc, Alameda, California). The coil mass was further packed with several $6 \mathrm{~mm}$ and $8 \mathrm{~mm}$ pushable coils (0.018 inches; Nester, Cook Medical, Bloomington, Indiana) to achieve adequate cross-sectional occlusion (-Fig. 5). A total of 21 coils were used to achieve cross-sectional occlusion and on the final venogram there was no further early filling of the left atrium. Manual compression was used to close the venotomy site at the conclusion of the procedure. The 


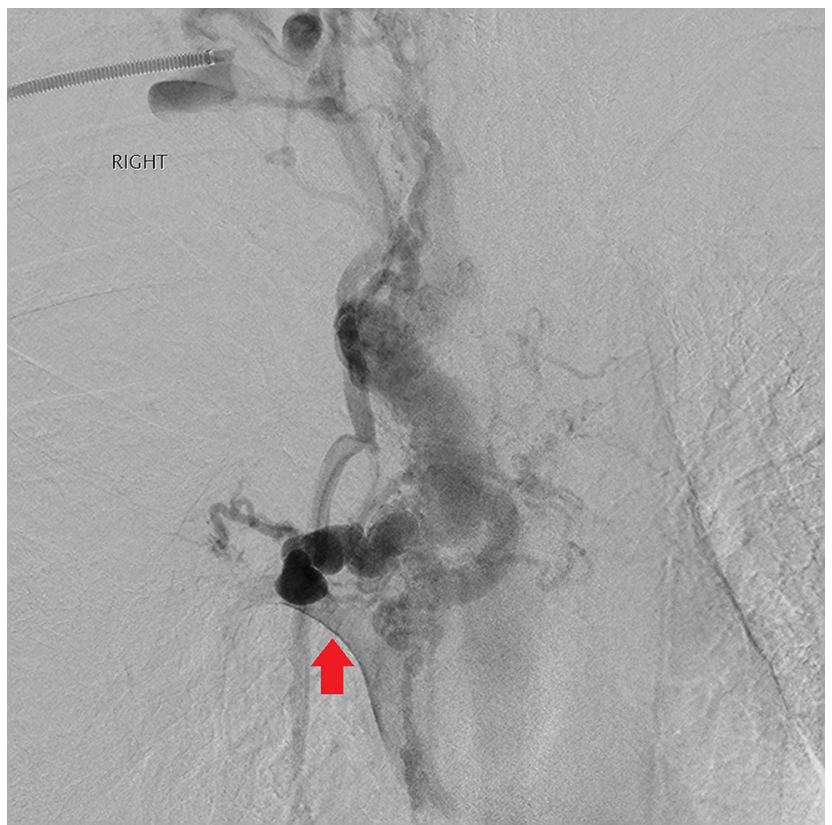

Fig. 4 Venogram from the right brachial sheath demonstrating superior vena cava (SVC) occlusion, development of tortuous mediastinal venous collaterals, and early filling of the left atrium (arrow).

patient re-started apixaban $5 \mathrm{mg}$ that evening and remains anticoagulated indefinitely. Follow-up venography and TTE with bubble study showed overall improvement when compared with the original study with recent venogram showing persistent mediastinal collateral veins (right-right shunts) but no early filling of the pulmonary veins/left atrium. The patient remains clinically silent form further cerebrovascular event at 12 months follow-up.

\section{Discussion}

Superior vena cava obstruction is an uncommon but recognized complication of CVADs; however, development of a subsequent right-to-left shunt is even rarer.-11 Options for management include surgical, endovascular, or medical therapy.

Surgical management offered to this patient included median sternotomy with bypass grafting from the brachiocephalic vein to right atrial appendage, and ligation of the shunt. This is efficacious; however, it is invasive with long recovery. Medical therapy would involve lifelong anticoagulation to decrease the rate of thromboembolism and reduce the risk of upper limb venous thrombosis as well as post-thrombotic syndrome. Endovascular treatment has two components. The first is to embolize the shunt/fistula and the second is to consider recannalization and stenting of the SVC. All three options are viable and carry different risk-benefit profiles that need to be considered.

The benefits of an embolization include less invasive access with the patient avoiding general anesthesia, with reduced potential for postoperative morbidity, short hospital stay, and faster recovery time than for surgery and associated reduction in direct and indirect costs. This should be

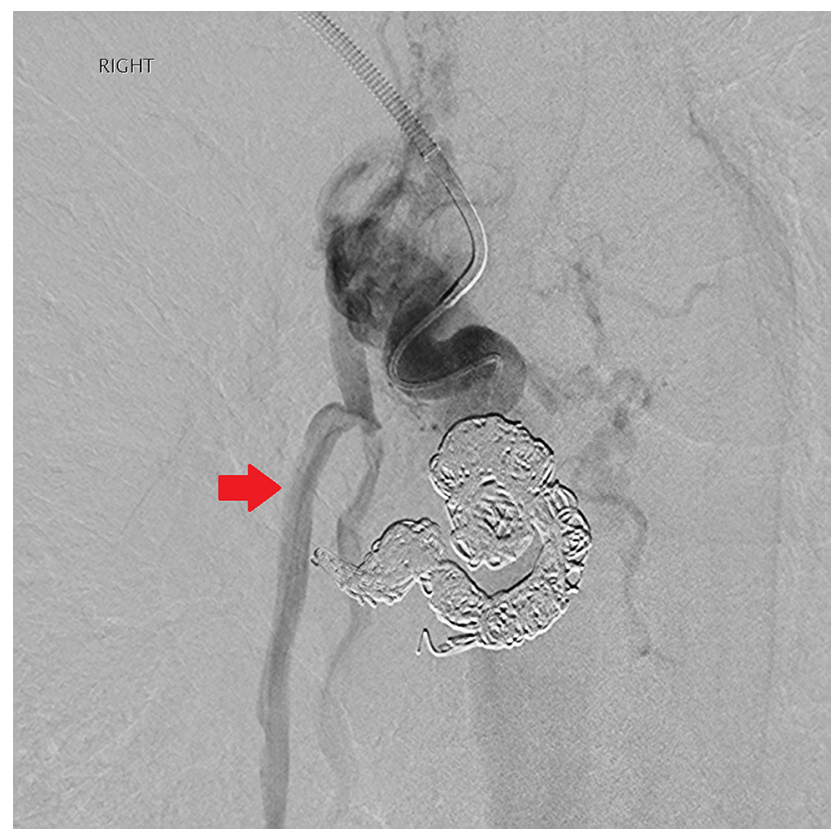

Fig. 5 Completion venogram showing coil mass placed into the main shunt with occlusion of flow. Right-to-right shunts (arrow) can also be seen filling the right atrium via the azygous system and other unnamed collaterals.

weighed against the risk of dislodging any preformed thrombus that may be in the channel at the time of embolization. This risk was managed by systemic anticoagulation during the procedure and the procedure was performed at a center with a neurointerventional service available should there be an unlikely need for cerebral clot retrieval, with this risk not ultimately materializing. In addition, long-term patency of the coil mass required consideration; however, for a low-flow system and with the use of numerous coils including fibered coils, the risk of this was considered negligible. The second component to consider with endovascular treatment is SVC recanalization. If the segment was short, wide, recent, or in the upper SVC segment, then this may be a favorable approach. This option is also ideal for patients with more severe symptoms of SVC syndrome. In our patient, the long and narrow occlusion coupled with it terminating at the right atrium required counseling of risks including mediastinal perforation and stent deployment in the right atrium. As the patient in this case had only mild symptoms of SVC syndrome it was agreed that this path would not be taken. If symptoms were to worsen, embolization alone would not preclude offering this in the future.

After the procedure, the patient remained on therapeutic-dose Non-vitamin Kantagonist oral anticoagulant (NOAC). Given that imaging showed multiple right-to-right shunts, it was hypothesized that a small residual right-to-left shunt may be beyond angiographic resolution so it was preferred to continue NOAC to keep the thromboembolism risk as low as possible in addition to the prevention of upper limb DVT and, thus, post-thrombotic syndrome.

Crucial to the success and minimization of CVAD thrombosis is the positioning of the tip; ideally placed in the 
inferior aspect of the SVC or at the cavoatrial junction. A previous study from Luciani et al of 145 patients demonstrated that patients with CVAD tips in the superior aspect of the SVC were found to develop catheter-related thrombosis 7 times higher than those placed more inferiorly. ${ }^{6}$ It has been shown that up to $40 \%$ of CVADs result in asymptomatic DVT and between 1 and 5\% in symptomatic DVTs. ${ }^{11,12}$

The presence of a CVAD is a documented iatrogenic cause of SVC obstruction. Once there is impediment of normal flow in the SVC, most patients would shunt venous return via the azygous and inferior vena caval pathway. In patients with occlusion covering the azygous tributary, venous blood would have to find less common collateral pathways to train to the IVC.7 Systemic to pulmonary venous collateral pathways can thus develop between the brachiocephalic and superior pulmonary veins via the bronchial venous plexuses around the airways, hilar vessels, and pleura. ${ }^{12}$ As these collateral pathways enlarge, a right-to-left shunt develops increasing the risk of stroke, cerebral abscess, and cardiac failure. ${ }^{4}$ Other common collateral pathways in SVC obstruction include the azygoshemiazygos (including intercostal and lumbar veins), internal and external mammary (including epigastric and superficial veins of the thorax), lateral thoracic (including lateral thoracic, thoracoepigastric, superficial circumflex, great saphenous, and femoral veins to IVC), and vertebral pathways (including brachiocephalic, vertebral, intercostal, lumbar, and sacral veins to collateralize to azygos/internal thoracic veins). ${ }^{12}$

In this case report, chronic SVC occlusion involving the azygous tributary resulted in formation of a shunt/fistula to the right superior pulmonary vein, resulting in paradoxical stroke. We highlight the efficacy of minimally invasive embolization of the shunt, thus reducing the potential for further paradoxical emboli in the future.

\section{Informed Consent}

Informed written consent was obtained from the patient in this case.

\section{Ethical Approval}

For case reports, specific ethical board approval is not required at our institute if the patient has provided consent.

\section{Funding}

None declared.

\section{Conflict of Interest}

None declared.

\section{References}

1 Wilson ES. Systemic to pulmonary venous communication in the superior vena caval syndrome. AJR Am J Roentgenol 1976;127(2):247-249

2 Cihangiroglu M, Lin BH, Dachman AH. Collateral pathways in superior vena caval obstruction as seen on CT. J Comput Assist Tomogr 2001;25(1):1-8

3 Madan AK, Allmon JC, Harding M, Cheng SS, Slakey DP. Dialysis access-induced superior vena cava syndrome. Am Surg 2002;68(10):904-906

4 Bashist B, Parisi A, Frager DH, Suster B. Abdominal CT findings when the superior vena cava, brachiocephalic vein, or subclavian vein is obstructed. AJR Am J Roentgenol 1996;167(6):1457-1463

5 Holemans JA, Howlett DC, Rankin SC. Case report: superior vena cava obstruction: unusual CT findings due to venous collaterals. ClinRadiol 1997;52(7):559-560

6 Nascimbene A, Angelini P. Superior vena cava thrombosis and paradoxical embolic stroke due to collateral drainage from the brachiocephalic vein to the left atrium. Tex Heart Inst J 2011;38(2):170-173

7 Muramatsu T, Miyamae T, Dohi Y. Collateral pathways observed by radionuclide superior cavography in 70 patients with superior vena caval obstruction. ClinNucl Med 1991;16(5):332-336

8 Avezbadalov A, Gutierrez C. Systemic-to-pulmonary venous shunt in a patient with non-Hodgkin lymphoma: a case report and review of the literature. Respir Med Case Rep 2015;15:9-11

9 Forauer AR, Theoharis CG, Dasika NL. Jugular vein catheter placement: histologic features and development of catheter-related (fibrin) sheaths in a swine model. Radiology 2006;240(2):427-434

10 Geerts W. Central venous catheter-related thrombosis. Hematology (Am SocHematolEduc Program) 2014; 2014(1):306-311

11 Grayet D, Ghaye B, Szapiro D, Dondelinger RF. Systemic-to-pulmonary venous shunt in superior vena cava obstruction revealed on dynamic helical CT. AJR Am J Roentgenol 2001;176(1):211-213

12 Kapur S, Paik E, Rezaei A, Vu DN. Where there is blood, there is a way: unusual collateral vessels in superior and inferior vena cava obstruction. Radiographics 2010;30(1):67-78 